поверхні [Текст]: тези доповіді до міжнародної конферениї «Моделювання $i$ дослідження стійкості систем» / П.І. Ванкевич, Л.Д. Величко, В.О. Фединець. K., 1997. - C. 22.

10. Ванкевич П.І. Теплообмін між контактними термоперетворювачами та дослідною поверхнею [Текст]: тези доповідей до міжнародного науково-практичного форуму «Теорія і практика АПК», 19-20 вересня, ЛДАУ, 2006. - Том 2. - C. 322-324.

11. Ванкевич П.І. Теплові величини - джерело інформації про стан технічних об єктів [Текст]: тези доп. П'ятого міжнар. симп. укр. інженерів-механіків у Львові: 16-18 травня. - Львів: КІНПАТРІ ЛТД, 2001. - С. 81.

12. Ванкевич П.І. Шляхи підвищення точності рухомих контактних термоперетворювачів [Текст] /
П.І. Ванкевич, О.М. Бурнаєв // Вимірювальна техніка та метрологія. - 2001. - Вип. 58. - С. 83-90.

13. Измерения в промышленности. Справ. изд. в 3-х кн. - Кн. 2. Способы измерения и аппаратура: Пер. с нем.[Под ред. П. Профоса]. - М.: Металлургия, 1990.$384 \mathrm{c}$.

14. Карслоу Г. Теплопроводность твёрдых тел [Текст] / Г. Карслоу, Д. Егер. - М.: Наука, 1964. - 487 c.

15. Хантер Н. Контактная задача качения жесткого ичлиндра по вязко-упругому полупространству [Текст] / Н. Хантер // Прикладная механика. - М.: Мир, 1961. - T. 28, № 4. - C. 146-153.

Рецензент: А.Ф. Обшта, д.т.н., проф., Навчальноконсультаційний центр, Національний транспортний університет, Львів.

РАСЧЕТ МЕТОДИЧЕСКОЙ ПОГРЕШНОСТИ ВРАЩАТЕЛЬНЫХ КОНТАКТНЫХ ТЕРМОПРЕОБРАЗОВАТЕЛЕЙ (ТП) ПРИ ИЗМЕРЕНИИ ТЕМПЕРАТУРЫ ДВИЖУЩИХСЯ ОБЬЕКТОВ

\author{
П.И. Ванкевич
}

Предложена методика математического расчета методической погрешности механических контактных термометров, что позволяет прогнозировать поведение показателей данного типа термометров в прочессе как постоянного, так и ииклического действия тепловой нагрузки. Полученные математические зависимости способны учитывать различные конфигурачии термопреобразователей и их физико-механические характеристики. Результатыл расчетов показывают, что полученное для конкретного случая измерительного прибора значение методической погрешности составляет доли процента от реальной температуры поверхности.

Ключевые слова: методическая погрешность, механические контактные термометры, тепловая нагрузка.

\title{
CALCULATION OF METHOD ERROR OF CONTACT-COUPLE THERMAL CONVERTERS WHEN MEASURING TEMPERATURES OF MOVING OBJECTS
}

$$
\text { P. Vankevych }
$$

The techique of method errors mathematical calculation of mechanical contact thermometers, which allows to predict the behavior of this type of thermometer hits in the process as a constant and cyclic thermal loading steps is offered. Obtained mathematical dependences are able to consider various configurations of thermocouples and their physical and mechanical properties. The calculation results show that obtained for case gauge the value of methodological error is fraction of a percent of actual surface temperature.

Keywords: method error, mechanical contact thermometers, heat load.

\section{УДК 621.396}

\section{С.В. Герасимов ${ }^{1}$, Е.С. Рощупкин ${ }^{1}$, Г.А. Федак ${ }^{2}$, Я.В. Бабий ${ }^{2}$}

${ }^{1}$ Научный центр Харьковського университета Воздушных Сил, г. Харьков.

${ }^{2}$ Академия сухопутных войск имени гетмана Петра Сагайдачного, г. Львов

\section{ОЦЕНКА ПАРАМЕТРОВ ДВИЖЕНИЯ МАНЕВРИРУЮЩИХ ВОЗДУШНЫХ ОБЪЕКТОВ В АКТИВНОЙ НЕКОГЕРЕНТНОЙ СИСТЕМЕ ПРИ ОБРАБОТКЕ ИНФОРМАЦИИ ОТ НЕСКОЛЬКИХ НЕРАВНОТОЧНЫХ ИСТОЧНИКОВ С РАЗНЫМ ТЕМПОМ ОБЗОРА ПРОСТРАНСТВА}

Предложен алгоритм определения параметров движения воздушного объекта в единой прямоугольной системе координат при обработке радиолокачионной информачии, получаемой системой с несинхронным обзором пространства от нескольких неравноточных источников с разным темпом обновления информачии.

Ключевые слова: радиолокачионная информачия, алгоритм, маневрирующие объекты 


\section{Постановка проблемы}

Идея интеграции радиолокационных систем различных ведомств вытекает из принятых Кабинетом Министров Украины распоряжения и постановления о «Государственной интегрированной информационной системе обеспечения управления подвижными объектами (связь, навигация, наблюдение) ». Использование информации единого радиолокационного поля позволит: обеспечить однозначное понимание воздушной обстановки в центрах управления воздушного движения и на пунктах управления видов Вооруженных Сил; исключить территориальное дублирование ведомственных информационных систем; обеспечить совместное использование радиоэлектронных средств; сократить типаж и унифицировать радиолокационные средства, комплексы средств автоматизации и связи.

\section{Анализ последних исследований}

\section{и публикаций}

В литературе, посвященной совместной обработке радиолокационной информации (РЛИ) от нескольких активных радиолокационных станций (РЛС) [1-8], вопросы определения параметров движения воздушного объекта (BO) в единой прямоугольной системе координат (ЕПСК) при обработке сигналов, получаемых некогерентной системой с несинхронным обзором пространства от нескольких неравноточных источников с разным темпом обновления информации, освещены недостаточно полно.

В работах [2-5] изучались вопросы обработки РЛИ от нескольких неравноточных РЛС на общем приемном пункте, однако несинхронность ее поступления, оценка параметров движения ВО и ошибки их измерения в ЕПСК рассмотрены не были. В работе [6] детально изучались вопросы оценки параметров движения ВО и ошибки их измерения по РЛИ одной РЛС. В работе [7] изучались вопросы оценки координат ВО и ошибки их измерения в ЕПСК при объединении результатов независимых первичных измерений в активной многопозиционной системе при синхронном обзоре пространства, оценка параметров движения ВО и ошибки их измерения в ЕПСК не рассматривались. В работах $[8,9]$ предложены алгоритмы определения параметров движения ВО, учитывающие несинхронность получения неравноточной информации отдельными пунктами активной многопозиционной системы, однако маневренные возможности ВО учтены не были.

Большинство из существующих систем обработки РЛИ от нескольких активных источников представляют собой пространственно-некогерент- ную многопозиционную систему с автономным приемом сигналов и объединением информации преимущественно на уровне единичных замеров. Для оценки параметров движения ВО, как правило, используется информация об измеренных первичных сферических координатах ВО относительно «приоритетного источника» (выбираемого исходя из взаимного расположения цели и пунктов системы, тактико-технических характеристик РЛС и других соображений). Информация, получаемая остальными РЛС системы, является вспомогательной и практически не используется в формировании оценки параметров движения ВО в ЕПСК, что позволяет отказаться от временной синхронизации позиций, существенно упрощает алгоритмы обработки и требования к вычислительным средствам, однако ведет к весомой потере точности измерений. При отсутствии РЛС с достаточной точностью измерения трех первичных координат в системе обычно используются результаты только высокоточных измерений соответствующих координат ВО двух РЛС (например, азимут и дальность одной станции, угол места от другой), как правило, разнесенных между собой на небольшое расстояние.

\section{Цель статьи}

Целью данной работы является синтез алгоритма определения параметров движения ВО в ЕПСК при обработке РЛИ, получаемой системой с несинхронным обзором пространства от нескольких неравноточных источников с разным темпом обновления информации, с учетом его маневренных возможностей.

\section{Изложение основного материала}

Пусть имеется система из $\mathrm{N}$ разнотипных неравноточных обзорных РЛС с разным темпом обзора пространства, разнесенных в пространстве на расстояние, существенно превышающее радиус пространственной корреляции принимаемых сигналов. За интервал времени завязки траектории $\mathrm{T}_{\text {завтр0 }}$ каждой $i$-й РЛС $\quad(\mathrm{i}=1 \ldots \mathrm{N})$ получено $\left(\mathrm{M}_{\mathrm{i}}+1\right)$ отметок-в моменты времени $\mathrm{t}_{\mathrm{j}} i$-й РЛС производится оценка сферических координат ВО $\hat{\overrightarrow{\mathrm{S}}}_{\mathrm{ij}}=\left\{\begin{array}{lll}\hat{\mathrm{R}}_{\mathrm{ij}} & \hat{\varepsilon}_{\mathrm{ij}} & \hat{\beta}_{\mathrm{ij}}\end{array}\right\}-$ наклонной дальности, угла места и азимута $\left(\mathrm{j}=0 . . \mathrm{M}_{\mathrm{i}}\right)$. Информация об отметках, оценках сферических координат и статистических характеристиках ошибок их измерения поступает на централизованный пункт обработки РЛИ в единой сетке времени (т.е. известен момент измерения координат ВО каждой РЛС, время задержки информации известно либо им можно пренебречь). Об оценках известно, что 


$$
\hat{S}_{i j}=S_{i j}^{*}+\Delta S_{i j}, i \in 1 \ldots N, j=0 . . M_{i}
$$

где $\mathrm{S}_{\mathrm{ij}}^{*}$ - вектор истинных значений сферических координат, $\Delta \mathrm{S}_{\mathrm{ij}}$ - вектор нормальных ошибок, обусловленных шумами, со следующими статистическими характеристиками

$$
\begin{gathered}
M\left[\Delta \mathrm{S}_{\mathrm{ijp}}\right\rfloor=0, \\
\mathrm{M}\left[\Delta \mathrm{S}_{\mathrm{ijp}} \Delta \mathrm{S}_{\mathrm{nmr}}\right]=\sigma^{2} \mathrm{Si}, \mathrm{j}, \mathrm{p} \delta_{\mathrm{i}, \mathrm{n}} \delta_{\mathrm{j}, \mathrm{m}} \delta_{\mathrm{p}, \mathrm{r}},
\end{gathered}
$$

где $\delta_{\mathrm{a}, \mathrm{b}}=\left\{\begin{array}{l}1, \mathrm{a}=\mathrm{b} \\ 0, \mathrm{a} \neq \mathrm{b}\end{array}-\right.$ символ Кронекера, $\mathrm{p}=1 . .3$.

Положим, что кривизна земной поверхности и рефракция радиоволн учтены. Тогда в ЕПСК $\overline{\overrightarrow{\mathrm{K}}}=\left\{\begin{array}{lll}\overline{\mathrm{x}} & \overline{\mathrm{y}} & \overline{\mathrm{z}}\end{array}\right\}$ - оценки прямоугольных координат ВО, связанные с оценками сферических координат относительно $i$-й РЛС системы функциональной зависимостью $\quad \hat{\overline{\mathrm{K}}}_{\mathrm{i} \mathrm{p}}=\hat{\mathrm{K}}_{\mathrm{i} \mathrm{j}}+\overline{\mathrm{K}} 0_{\mathrm{ip}}=\varphi\left(\hat{\overrightarrow{\mathrm{S}}}_{\mathrm{ij}}\right)+\overline{\mathrm{K}} 0_{\mathrm{ip}}$, имеют вид (рис. 1):

$$
\left\{\begin{array}{l}
\hat{\bar{x}}_{i j}=\hat{x}_{i j}+\bar{x}_{0 i}=\hat{R}_{i j} \cdot \cos \hat{\varepsilon}_{i j} \cdot \cos \hat{\beta}_{i j}+\bar{x}_{0 i} \\
\hat{\bar{y}}_{i j}=\hat{y}_{i j}+\bar{y}_{0 i}=\hat{R}_{i j} \cdot \sin \hat{\varepsilon}_{i j}+\bar{y}_{0 i} \\
\hat{\bar{z}}_{i j}=\hat{z}_{i j}+\bar{z}_{0 i}=\hat{R}_{i j} \cdot \cos \hat{\varepsilon}_{i j} \cdot \sin \hat{\beta}_{i j}+\bar{z}_{0 i}
\end{array},\right.
$$

где $\hat{\mathrm{K}}_{\mathrm{i} \text { jp }} \quad-\quad$ оценки соответствующих прямоугольных координат ВО относительно $i$-й РЛС в момент времени $\mathrm{t}_{\mathrm{ij}}, \overline{\mathrm{K}} 0_{\mathrm{i}}$ - вектор известных координат $i$-й РЛС системы в ЕПСК, $\mathrm{p}=1 . .3$.

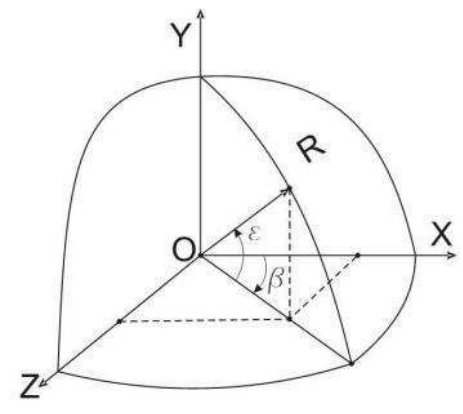

Puc. 1. Связь координат цели

В качестве примера на рис. 2 схематически представлен один из вариантов поступления во времени на централизованный пункт обработки информации отметок от трех источников об одном ВО. Для $i$-й РЛС временные интервалы $\Delta \mathrm{t}_{\mathrm{ij}}-$ задержки поступления ј отметки РЛИ относительно начального момента поступления информации о ВО
( $\mathrm{j}=0$ ) или предыдущего момента получения информации о ВО от той же РЛС $(\mathrm{j} \neq 0)$.

Необходимо получить алгоритм определения параметров движения ВО в ЕПСК при обработке РЛИ, получаемой системой с несинхронным обзором пространства от нескольких неравноточных источников с разным темпом обновления информации.

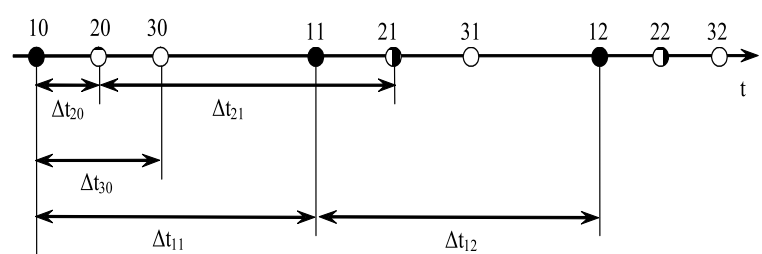

- отметки от первой РЛС
$\ominus$ - отметки от второй РЛС
- отметки от третьей РЛС

Puc. 2. Поступление во времени информации о ВО

На ВО в процессе полета воздействуют случайные факторы, влияющие на параметры его траектории. Можно показать, что при измерении координат маневрирующего ВО обзорными РЛС аппроксимация траектории его движения полиномом выше степени $\mathrm{w}_{\max }=2$ является нецелесообразной $[5,8,9]$. Модель траектории движения ВО по соответствующей прямоугольной координате на небольшом промежутке времени $\mathrm{T}_{\text {завтр0 }}$ порядка 10-25 секунд (1-5 периодов обзора большинства существующих обзорных РЛС) в ЕПСК в большинстве случаев достаточно точно может быть записана следующим образом:

$$
\left.\begin{array}{l}
x(t)=x_{0}+\vartheta_{x} \cdot t+\frac{a_{x} \cdot t^{2}}{2}+\Phi_{x}(t) \\
y(t)=y_{0}+\vartheta_{y} \cdot t+\frac{a_{y} \cdot t^{2}}{2}+\Phi_{y}(t) \\
z(t)=z_{0}+\vartheta_{z} \cdot t+\frac{a_{z} \cdot t^{2}}{2}+\Phi_{z}(t)
\end{array}\right\}
$$

или в общем виде

$k_{k}(t)=k_{0 k}+\vartheta_{k} t+\frac{a_{k} t^{2}}{2}+N_{k}(t)=\sum_{w=0}^{w a n p} k_{w} \frac{t^{w}}{w !}+\Phi_{k}(t)$ где $\mathrm{k}_{0 \mathrm{k}}$ - начальное значение соответствующей координаты, $\mathrm{k}_{1 \mathrm{k}}=\vartheta_{\mathrm{k}}$ и $\mathrm{k}_{2 \mathrm{k}}=\mathrm{a}_{\mathrm{k}}-$ проекции начальной скорости и ускорения соответственно, 
$\Phi_{\mathrm{k}}(\mathrm{t})$ - составляющая случайных факторов, искажающих траекторию движения ВО по соответствующей координате, $\mathrm{w}=0 \ldots \mathrm{w}_{\text {aпр }}$, $1 \leq \mathrm{w}_{\text {апр }} \leq 2$. При этом предположим, что за интервал времени, равный темпу обновления информации о $\mathrm{BO} \mathrm{T}_{\text {обн }}$ (переменный в общем случае),

$$
\begin{gathered}
\left|\mathrm{M}\left[\Phi_{\mathrm{k}}\right]\right|<<\left|\mathrm{k}_{\mathrm{w} \text { max }}\right|, \\
\mathrm{M}\left[\Phi_{\mathrm{k}}-\mathrm{M}\left[\Phi_{\mathrm{k}}\right]\right]^{2}+\mathrm{M}\left[\Phi_{\mathrm{k}}\right]^{2}<\left[\mathrm{k}_{\mathrm{w} \text { max }}\right]^{2}, \\
\mathrm{M}\left[\Phi_{\mathrm{k}}-\mathrm{M}\left[\Phi_{\mathrm{k}}\right]\right]^{2}+\mathrm{M}\left[\Phi_{\mathrm{k}}\right]^{2}<\sigma_{\mathrm{k}}{ }^{2}, \\
\sigma_{\mathrm{k}}{ }^{2}<\left[\mathrm{k}_{\mathrm{w} \max } \frac{\mathrm{T}_{\text {обн }}{ }_{\mathrm{w} \mathrm{max}}}{\mathrm{w}_{\text {max }} !}\right]^{2},
\end{gathered}
$$

где $\sigma_{\mathrm{k}}^{2}$ - результирующая дисперсия оценки соответствующей координаты ВО. В работе [9] получены соотношения для плотностей вероятности оценок прямоугольных координат ВО относительно РЛС (3) и числовых характеристик их параметров:

$$
\begin{aligned}
& \operatorname{fx}(\hat{\mathrm{R}}, \hat{\varepsilon}, \hat{\beta})=\frac{\left(\sqrt{(2 \pi)^{3}} \sigma_{\mathrm{R}} \sigma_{\varepsilon} \sigma_{\beta}\right)^{-1}}{\sqrt{\left[1-\cos ^{2}(\hat{\varepsilon})\right]\left[1-\cos ^{2}(\hat{\beta})\right]}} \times \\
& \times \exp \left\{-\left[\begin{array}{l}
\frac{\left(\hat{\mathrm{R}}-\mathrm{R}^{*}\right)^{2}}{2 \sigma^{2} \mathrm{R}}+\frac{\left(\arccos [\cos (\hat{\varepsilon})]-\varepsilon^{*}\right)^{2}}{2 \sigma^{2} \varepsilon}+ \\
+\frac{\left(\arccos [\cos (\hat{\beta})]-\beta^{*}\right)^{2}}{2 \sigma^{2} \beta}
\end{array}\right]\right\} \\
& \operatorname{fy}(\hat{\mathrm{R}}, \hat{\varepsilon}, \hat{\beta})=\frac{\left(2 \pi \sigma_{\mathrm{R}} \sigma_{\varepsilon}\right)^{-1}}{\sqrt{1-\sin ^{2}(\hat{\varepsilon})}} \times \\
& \times \exp \left\{-\left[\frac{\left(\hat{\mathrm{R}}-\mathrm{R}^{*}\right)^{2}}{2 \sigma^{2} \mathrm{R}}+\frac{\left(\arcsin [\sin (\hat{\varepsilon})]-\varepsilon^{*}\right)^{2}}{2 \sigma_{\varepsilon}^{2}}\right]\right\} \\
& \mathrm{z}(\hat{\mathrm{R}}, \hat{\varepsilon}, \hat{\beta})=\frac{\left(\sqrt{(2 \pi)^{3}} \sigma_{\mathrm{R}} \sigma_{\varepsilon} \sigma_{\beta}\right)^{-1}}{\sqrt{\left[1-\cos ^{2}(\hat{\varepsilon})\right] \cdot\left[1-\sin ^{2}(\hat{\beta})\right]}} \times \\
& \times \exp \left\{-\left[\begin{array}{l}
\frac{\left(\hat{\mathrm{R}}-\mathrm{R}^{*}\right)^{2}}{2 \sigma^{2} \mathrm{R}}+\frac{\left(\arccos [\cos (\hat{\varepsilon})]-\varepsilon^{*}\right)^{2}}{2 \sigma_{\varepsilon}^{2}}+ \\
+\frac{\left(\arcsin [\sin (\hat{\beta})]-\beta^{*}\right)^{2}}{2 \sigma^{2} \beta}
\end{array}\right]\right\}
\end{aligned}
$$

$$
\left\{\begin{array}{c}
M\left[\hat{x}_{i j}\right]=x_{i j}{ }^{*} \exp \left\{-\left(\sigma_{\varepsilon i j}^{2}+\sigma_{\beta i j}^{2}\right) / 2\right\} \\
M\left[\hat{y}_{i j}\right]=y_{i j}{ }^{*} \exp \left\{-\sigma_{\varepsilon i j}^{2} / 2\right\} \\
M\left[\hat{z}_{i j}\right]=z_{i j}{ }^{*} \exp \left\{-\left(\sigma_{\varepsilon i j}^{2}+\sigma_{\beta i j}^{2}\right) / 2\right.
\end{array}\right\}
$$

$$
\begin{aligned}
\sigma_{\hat{\mathrm{x}} \mathrm{ij}}^{2}= & \frac{\left(\mathrm{R}_{\mathrm{ij}}^{*}\right)^{2}+\sigma_{\mathrm{Rij}}^{2}\left[1+\cos \left(2 \varepsilon_{\mathrm{ij}}^{*}\right) \exp \left(-2 \sigma_{\varepsilon \mathrm{ij}}^{2}\right\}\right] \times}{4} \\
& \times\left[1+\cos \left(2 \beta_{\mathrm{ij}}^{*}\right) \exp \left\{-2 \sigma_{\beta \mathrm{ij}}^{2}\right\}\right]- \\
& -\left[\mathrm{x}_{\mathrm{ij}}^{*}\right]^{2} \exp \left\{-\left(\sigma_{\varepsilon \mathrm{ij}}^{2}+\sigma_{\beta \mathrm{ij}}^{2}\right)\right\} \\
\sigma_{\hat{\mathrm{y}} \mathrm{ij}}^{2}= & \frac{\left(\mathrm{R}_{\mathrm{ij}}^{*}\right)^{2}+\sigma_{\mathrm{Rij}}^{2}\left[1-\cos \left(2 \varepsilon_{\mathrm{ij}}^{*}\right) \exp \left(-2 \sigma_{\varepsilon \mathrm{ij}}^{2}\right\}\right]-}{2} \\
& -\left[\mathrm{y}_{\mathrm{ij}}{ }^{2}\right]^{2} \exp \left\{-\sigma_{\varepsilon \mathrm{ij}}^{2}\right\} \\
\sigma_{\hat{\mathrm{z}} \mathrm{ij}}^{2}= & \left(\mathrm{R}_{\mathrm{ij}}^{*}\right)^{2}+\sigma_{\mathrm{Rij}}^{2}\left[1+\cos \left(2 \varepsilon_{\mathrm{ij}}^{*}\right) \exp \left\{-2 \sigma_{\varepsilon \mathrm{ij}}^{2}\right\}\right] \times \\
& \times\left[1-\cos \left(2 \beta_{\mathrm{ij}}^{*}\right) \exp \left\{-2 \sigma_{\beta \mathrm{ij}}^{2}\right\}\right]- \\
& -\left[\mathrm{z}_{\mathrm{ij}}{ }^{2}\right]^{2} \exp \left\{-\left(\sigma_{\varepsilon \mathrm{ij}}^{2}+\sigma_{\beta \mathrm{ij}}^{2}\right)\right\}
\end{aligned}
$$

Показано, что нелинейная зависимость прямоугольных координат цели от ее угловых сферических координат при наличии нормальных флуктуационных ошибок измерения со статистическими характеристиками (2) приводит к смещению оценок прямоугольных координат цели (7), систематические ошибки оценок прямоугольных координат цели $\Delta \hat{\mathrm{K}}_{\mathrm{ij}}^{\mathrm{s}}=\mathrm{K}_{\mathrm{ij}}^{*}-\mathrm{M}\left[\hat{\mathrm{K}}_{\mathrm{ij}}\right]$ описываются выражениями

$$
\left\{\begin{array}{c}
\Delta \hat{\mathrm{x}}_{\mathrm{ij}}^{\mathrm{s}}=\mathrm{x}_{\mathrm{ij}}^{*} \cdot\left[1-\exp \left\{-\left(\sigma_{\varepsilon \mathrm{ij}}^{2}+\sigma_{\beta \mathrm{ij}}^{2}\right) / 2\right\}\right] \\
\Delta \hat{y}_{\mathrm{ij}}^{\mathrm{s}}=\mathrm{y}_{\mathrm{ij}}^{*} \cdot\left[1-\exp \left\{-\sigma_{\varepsilon \mathrm{ij}}^{2} / 2\right\}\right] \\
\Delta \hat{\mathrm{z}}_{\mathrm{ij}}^{\mathrm{s}}=\mathrm{z}_{\mathrm{ij}}^{*} \cdot\left[1-\exp \left\{\left(\sigma_{\varepsilon \mathrm{ij}}^{2}+\sigma_{\beta \mathrm{ij}}^{2}\right) / 2\right\}\right]
\end{array} .\right.
$$

При пренебрежении систематическими ошибками измерения координат (9), зная оценки сферических координат ВО $\hat{\overrightarrow{\mathbf{S}}}_{\mathbf{i j}}=\left\{\begin{array}{lll}\hat{R}_{i j} & \hat{\varepsilon}_{i j} & \hat{\boldsymbol{\beta}}_{i j}\end{array}\right\}$ и статистические характеристики ошибок их измерения отдельными РЛС (2), можно получить статистические характеристики ошибок измерения прямоугольных координат $\sigma_{\mathrm{k}}^{2} \mathrm{j} \quad$ (8). Это дает возможность перейти от неравноточных измерений к равноточным - к обработке результатов измерений c «весом» $\mathrm{P}_{\mathrm{q}}=\sigma_{\mathrm{q}}{ }^{-2}$, где $\sigma_{\mathrm{q}}^{-2}-$ дисперсия ошибки соответствующего измерения, $\mathrm{q}=1 . . \Sigma$, 
$\Sigma=\sum_{\mathrm{i}=1}^{\mathrm{N}}\left(\mathrm{M}_{\mathrm{i}}+1\right)$ - общее число отметок от ВО. В случае, когда

$$
\Sigma=\sum_{\mathrm{i}=1}^{\mathrm{N}}\left(\mathrm{M}_{\mathrm{i}}+1\right)>\mathrm{w}_{\mathrm{anp}}
$$

уравнение параметров его движения по соответствующей координате можно записать следующим образом $[8,9]$ :

$$
\mathrm{T}^{\mathrm{T}} \cdot \mathrm{P} \cdot \mathrm{T} \cdot \overline{\mathrm{K}} \mathrm{c}=\mathrm{T}^{\mathrm{T}} \cdot \mathrm{P} \cdot \mathrm{K}
$$

где при $\mathrm{w}_{\text {апр }}=\mathrm{w}_{\text {max }}=2$;

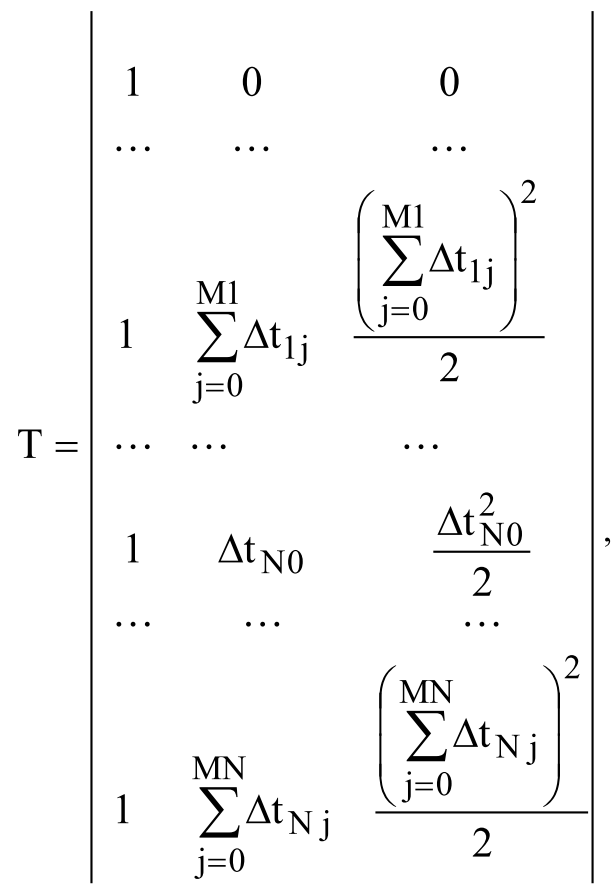

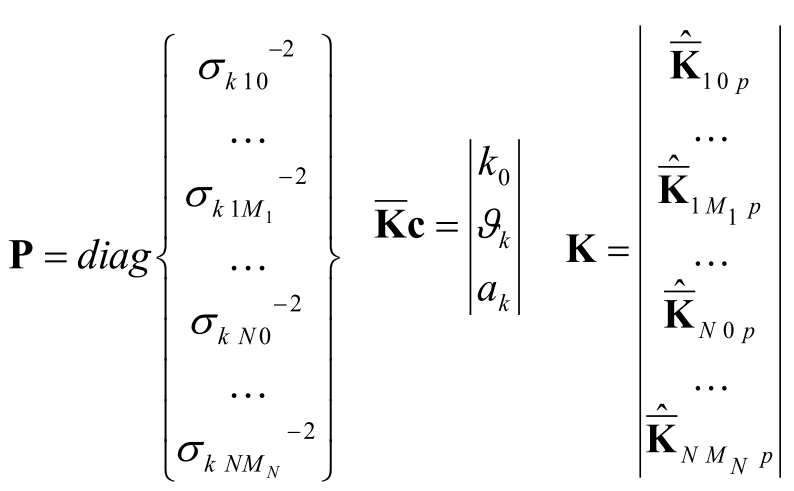

Условно назовем вышеприведенные матрицы: T - «временная», Р - «весовая», $\overline{\mathrm{K}} \mathrm{c}$ - «параметров движения», $\mathrm{K}$ - «координат ЕПСК».

В общем случае $\sigma_{\mathrm{k} \text { a,b }} \neq \sigma_{\mathrm{k}} \mathrm{c}, \mathrm{d}$.

Вектор оценок параметров движения ВО однозначно определяется по методу наименьших квадратов из матричного уравнения

$$
\hat{\bar{K}} \mathrm{c}=\left[\mathrm{T}^{\mathrm{T}} \cdot \mathrm{P} \cdot \mathrm{T}\right]^{-1} \cdot \mathrm{T}^{\mathrm{T}} \cdot \mathrm{P} \cdot \mathrm{K} .
$$

В предположении о малости систематических ошибок измерения координат (9) и справедливости соотношений (5) можно полагать, что вектор оценок параметров траектории $\quad \overline{\mathrm{K}} \mathrm{c}=\left\{\begin{array}{lll}\mathrm{k}_{0} & \vartheta_{\mathrm{k}} & \mathrm{a}_{\mathrm{k}}\end{array}\right\}^{\mathrm{T}}$ является асимптотически несмещенным, а дисперсии измерения соответствующих параметров определяются выражением

$$
\sigma_{\overline{\mathrm{K}} \mathrm{w}}^{2} \approx \sum_{\mathrm{q}=1}^{\Sigma} \Omega_{\mathrm{wq}}^{2} \cdot \sigma_{\mathrm{kq}}^{2}
$$

где

$$
\begin{gathered}
\Omega=\left[\mathrm{T}^{\mathrm{T}} \cdot \mathrm{P} \cdot \mathrm{T}\right]^{-1} \cdot \mathrm{T}^{\mathrm{T}} \cdot \mathrm{P}, \\
\mathrm{w}=0 . . \mathrm{w}_{\text {aпр }}=\mathrm{w}_{\max }=2, \mathrm{q}=1 . . \Sigma .
\end{gathered}
$$

Сглаженное значение координат ВО определяется выражением

$$
\widetilde{\mathrm{K}}=\mathrm{T} \cdot \hat{\overline{\mathrm{K}}} \mathrm{c} .
$$

Введем в рассмотрение «плоскость движения ВО», под которой будем понимать некоторую плоскость $v 0 \zeta$ с результирующей матрицей поворота W (матрицей направляющих косинусов) относительно единой прямоугольной системы координат [10]

$$
\left\{\begin{array}{lll}
\vartheta & \psi & \zeta
\end{array}\right\}^{\mathrm{T}}=\mathrm{W} \cdot\left\{\begin{array}{lll}
\mathrm{x} & \mathrm{y} & \mathrm{z}
\end{array}\right\}^{\mathrm{T}},
$$

для которой в течение интервала времени $\mathrm{T}_{\text {завтр0 }}$ справедливо неравенство

$$
\begin{aligned}
& \mathrm{L}_{\mathrm{q}}{ }^{2} \leq \sigma^{2}+\sum_{\mathrm{k}} \mathrm{M}\left[\Phi_{\mathrm{k}}-\mathrm{M}\left[\Phi_{\mathrm{k}}\right]\right]^{2}+\mathrm{M}\left[\Phi_{\mathrm{k}}\right]^{2}, \\
& \text { где } \mathrm{L}_{\mathrm{q}}-\text { удаление точек сглаженной }
\end{aligned}
$$

траектории ВО от плоскости его движения, $\sum_{\mathrm{q}=1}^{\Sigma} \mathrm{L}_{\mathrm{q}}{ }^{2} \rightarrow \min , \sigma^{2}=\sigma_{\mathrm{x}}{ }^{2}+\sigma_{\mathrm{y}}{ }^{2}+\sigma_{\mathrm{z}}{ }^{2}-$ результирующая дисперсия ошибки измерения прямоугольных координат ВО. Направление оси $v$ совпадает с направлением проекции оценки вектора скорости ВО $\hat{\vec{\vartheta}}$ на плоскость $v 0 \zeta$ в начальный момент

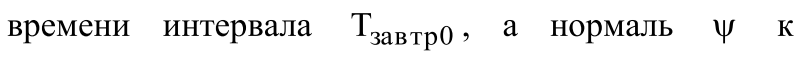
плоскости $v 0 \zeta$ образует правую систему координат $\vartheta \psi \zeta$.

Под минимальным радиусом разворота ВО

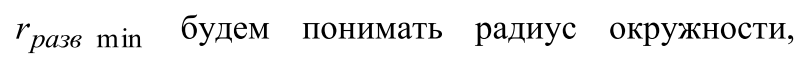
лежащей в плоскости $v 0 \zeta$, по дуге которой, исходя из допустимых перегрузок (максимального нормального ускорения $\left.\mathrm{a}_{\text {normmax }}=\mathrm{n}_{\max } \cdot \mathrm{g}\right)$, возможен маневр ВО

$$
r_{\text {разв } \min }=|\hat{\vec{\vartheta}}|^{2}\left(n_{\max } \cdot g\right)^{-1}
$$


где $|\hat{\vec{\vartheta}}|=\sqrt{\sum_{\mathrm{k}}\left(\vartheta_{\mathrm{k}}+\frac{\mathrm{a}_{\mathrm{k}} \mathrm{T}_{\text {зав тр } 0}}{2}\right)^{2}}-$ средняя скорость ВО за интервал времени завязки траектории.

При априорно известных маневренных возможностях сопровождаемого ВО $\mathrm{a}_{\text {norm } \max }=\mathrm{n}_{\max } \cdot \mathrm{g}$ (т.е. $\Theta \neq 0$, где $\Theta-$ признак наличия информации об маневренных возможностях ВО $\mathrm{a}_{\text {norm } \max }$ ), для плоскости движения ВО v0弓 могут быть рассчитаны $[7,9]$ :

- минимальный радиус разворота (17);

- период $\mathrm{T}_{\text {вр }}$ и угловая скорость $\omega_{\text {вр }}$ вращения

$\mathrm{T}_{\text {вр }}=2 \cdot \pi \cdot|\hat{\vec{\vartheta}}| \cdot\left(\mathrm{n}_{\max } \cdot \mathrm{g}\right)^{-1}, \quad \omega_{\text {вр }}=\mathrm{n}_{\max } \cdot \mathrm{g} \cdot|\hat{\vec{\vartheta}}|^{-1} ;$

- минимальная длительность интервала $T_{\text {зав тр } \min }$

$$
\mathrm{T}_{\text {зав тртіn }}=\frac{\mathrm{T}_{\text {вр }}}{4},
$$

в течение которого ориентация вектора скорости ВО в плоскости движения может поменяться на $90^{\circ}$ при совершении маневра;

- число отметок от ВО $\Sigma_{\min }$, попадающих в интервал времени $\mathrm{T}_{\text {завтртіn }}$, для которых выполняется условие

$$
\Sigma_{\min }=\sum_{\mathrm{i}=1}^{\mathrm{N}} \mathrm{M}_{\mathrm{i}} \text { при } \Delta \mathrm{t}_{\mathrm{ij}} \leq \mathrm{T}_{\text {зав тр min }}
$$

- математические ожидания и дисперсии отклонений координат ВО при совершении маневра в плоскости движения по дуге окружности с ради-

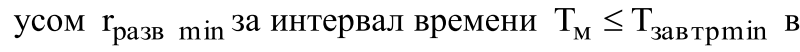
предположении, что в течение данного интервала времени вероятность совершения маневра распределена по равномерному закону («равновероятный маневр»):

$$
\begin{aligned}
& \left|\mathrm{M}\left[\Delta \mathrm{v}_{\text {max }}\right]\right|=\int_{0}^{\mathrm{TM}} \frac{|\hat{\vec{\vartheta}}| \mathrm{t}-\mathrm{r}_{\text {разв } \min } \sin \left(\omega_{\mathrm{Bp}} \mathrm{t}\right)}{\mathrm{T}_{\mathrm{M}}} \mathrm{dt}= \\
& =|\hat{\vec{\vartheta}}| \frac{\left(\mathrm{n}_{\text {max }} \mathrm{gT} \mathrm{T}_{\mathrm{M}}\right)^{2}+2|\hat{\vec{\vartheta}}|^{2}\left(1-\cos \left(\phi_{\mathrm{M}}\right)\right)}{2\left(\mathrm{n}_{\max } \mathrm{g}\right)^{2} \mathrm{~T}_{\mathrm{M}}}
\end{aligned}
$$

$$
\begin{aligned}
& \sigma_{\Delta v \max }^{2}=\frac{|\hat{\vec{\vartheta}}|^{2}}{12\left(\mathrm{n}_{\max } \mathrm{g}\right)^{4} \mathrm{~T}_{\mathrm{M}}{ }^{2}} \times \\
& \times\left(\left(\mathrm{n}_{\max } g \mathrm{~T}_{\mathrm{M}}\right)^{4}-12|\hat{\vec{\vartheta}}|^{4}\left(1-\cos \left(\phi_{\mathrm{M}}\right)\right)^{2}+\right.
\end{aligned}
$$

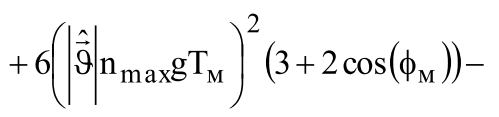

$$
\begin{aligned}
& \left.-6|\hat{\vec{\vartheta}}|^{3} \mathrm{n}_{\text {max }_{M}} \mathrm{gT}_{\mathrm{M}} \sin \left(\phi_{\mathrm{M}}\right)\left(4+\cos \left(\phi_{\mathrm{M}}\right)\right)\right) \\
& \left|\mathrm{M}\left[\Delta \zeta_{\max }\right]\right|=\int_{0}^{\mathrm{T}_{\mathrm{M}}} \frac{\mathrm{r}_{\mathrm{pa} \min }\left(1-\cos \left(\omega_{\mathrm{Bp}} \mathrm{t}\right)\right)}{\mathrm{T}_{\mathrm{M}}} \mathrm{dt}= \\
& =\frac{1}{\left(\mathrm{n}_{\max } \mathrm{g}\right)^{2} \mathrm{~T}_{\mathrm{M}}} \cdot|\overrightarrow{\hat{\vartheta}}|^{2}\left(\mathrm{n}_{\max } \mathrm{gT}_{\mathrm{M}}-|\hat{\vec{\vartheta}}| \sin \left(\phi_{\mathrm{M}}\right)\right)^{\prime} \\
& \sigma_{\Delta \zeta \text { max }}{ }^{2}=|\hat{\vec{\vartheta}}|^{4} \frac{1}{2\left(\mathrm{n}_{\max } \mathrm{g}\right)^{4} \mathrm{~T}_{\mathrm{M}}{ }^{2}} \cdot\left(\left(\mathrm{n}_{\max } \mathrm{gT}_{\mathrm{M}}\right)^{2}+\right.
\end{aligned}
$$

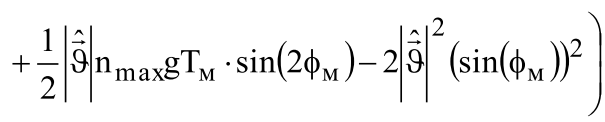

Для большинства современных типов ВО (кроме летящих по баллистической траектории) характерно, что существенное изменение параметров их движения возможно лишь в поперечном (перпендикулярном вектору скорости) направлении:

$$
\mathrm{a}_{\text {norm }}>\mathrm{a}_{\text {prod }}
$$

где $a_{\text {prod }}$ - продольная составляющая ускорения ВО, совпадающая по направлению с вектором скорости.

Таким образом, можно полагать, что отличие от нуля модуля оценки текущего ускорения ВО

$$
\left|\hat{\overrightarrow{\mathrm{a}}}_{\text {тек }}\right|=\sqrt{\sum_{\mathrm{k}}\left(\mathrm{a}_{\mathrm{k}}\right)^{2}} \cong\left|\mathrm{a}_{\text {norm }}\right| \geq \theta \cdot \sigma_{\mathrm{a}} \neq 0
$$

свидетельствует о маневре ВО в плоскости движения, где $\theta$ - некоторая величина, зависящая от вероятности правильного обнаружения маневра $[5,9]$.

Подставляя в (17)-(18) текущие значения оценок векторов скорости и ускорения ( $\hat{\vec{v}}_{\text {тек }}$ и $\hat{\vec{a}}_{\text {тек }}$ ), можно оценить текущие значения $\hat{\mathrm{r}}_{\text {разв тек }}, \hat{\mathrm{T}}_{\mathrm{Bp}}$ тек и $\hat{\omega}_{\text {вр тек }}$, а следовательно, из (19) - (24) - $\hat{\mathrm{T}}_{\text {завтртек }}$, $\hat{\Sigma}_{\text {тек }}$ (целесообразное для использования в (12) число отметок от ВО, попадающих в интервал времени $\left.\hat{\mathrm{T}}_{\text {завтртек }}\left(\Sigma_{\min } \leq \hat{\Sigma}_{\text {тек }} \leq \Sigma\right)\right),\left|\mathrm{M}\left[\Delta \mathrm{v}_{\text {тек }}\right]\right|$, $\sigma_{\Delta \text { v тек }}^{2},\left|\mathrm{M}\left[\Delta \zeta_{\text {тек }}\right]\right|$ и $\sigma_{\Delta \zeta \text { тек }}^{2}$. 
В ЕПСК статистические характеристики отклонений координат ВО при совершении «равновероятного маневра» определяются соотношениями

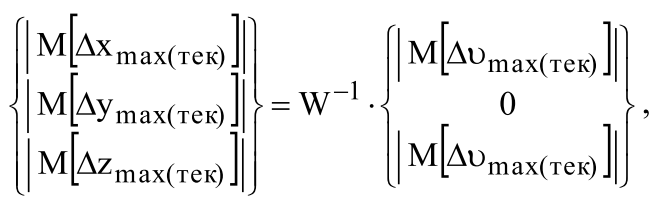

$$
\left\{\begin{array}{l}
\sigma_{\Delta \mathrm{x}} \max (\text { тек) } \\
\sigma_{\Delta \mathrm{y} \max \left(\text { тек }{ }_{2}^{2}\right.}^{2} \\
\sigma_{\Delta \mathrm{z} \max (\text { тек })}^{2}
\end{array}\right\}=\mathrm{W}^{-1} \cdot\left\{\begin{array}{c}
\sigma_{\Delta \mathrm{U}} \max (\text { тек })^{2} \\
0 \\
\sigma_{\Delta \zeta \max (\text { тек })}{ }^{2}
\end{array}\right\} .
$$

Упрощенный алгоритм оценки параметров движения ВО приведен на рис. 3.

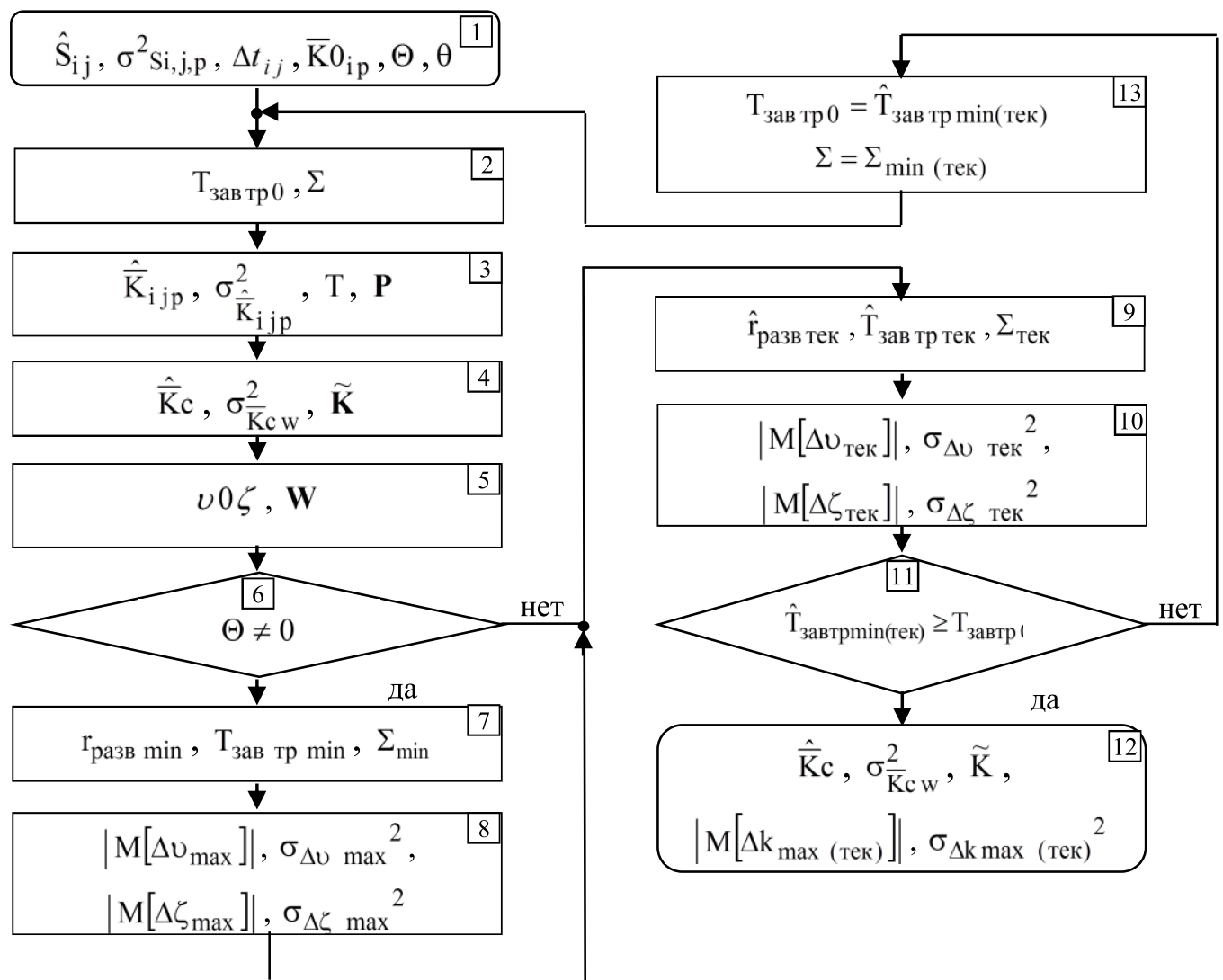

Puc. 3. Упрощенный алгоритм оценки параметров движения ВО $\left(w_{\text {mã̃ }}=2<\Sigma_{\min (\text { ə̊åe })}\right)$

Кратко раскроем содержание основных операций алгоритма.

Блок 1. В качестве исходных данных используются: оценки сферических координат ВО и статистические характеристики ошибок их измерения. Известны: временные интервалы получения оценок, координаты приемных пунктов, априорная информация о предполагаемых маневренных возможностях ВО, задана вероятность правильного обнаружения маневра.

Блок 2. По имеющимся данным определяется «первоначальный» временной интервал завязки траектории и число используемых отметок (оценок координат).

Блок 3. Проводится пересчет оценок сферических координат ВО относительно приемных пунктов в оценки прямоугольных координат в ЕПСК. Определяются статистические характеристики оценок прямоугольных координат (они используются для формирования «весовой» матрицы), формируется «временная» матрица.

Блок 4. Проводится оценка «параметров движения», рассчитываются статистические характеристики ошибок их определения. Определяется сглаженное значение координат ВО.

Блок 5. Задается «плоскость движения ВО»и и матрица ее направляющих косинусов относительно ЕПСК.

Блок 6. При наличии априорной информации о предполагаемых маневренных возможностях ВО проводится расчет параметров «равновероятного маневра» (блоки 7, 8).

Блок 7. Определяются «минимальные» радиус разворота и интервал времени, в течение которого ориентация вектора скорости ВО в плоскости движения может поменяться на $90^{\circ}$, при совершении маневра с максимальной перегрузкой. Определяется количество отметок (оценок координат ВО), 
которые попадают в данный «минимальный» временной интервал.

Блок 8. Определяются статистические характеристики «равновероятного маневра» за «минимальный» временной интервал в «плоскости движения ВО».

Блок 9. Используя текущие значения оценок векторов скорости и ускорения, проводится оценка «текущих» значений радиуса разворота и интервала времени, в течение которого ориентация вектора скорости ВО в плоскости движения может поменяться на $90^{\circ}$. Определяется количество отметок (оценок координат ВО), которые попадают в данный «текущий» временной интервал.

Блок 10. Определяются статистические характеристики «равновероятного маневра» за «текущий» временной интервал в «плоскости движения ВО».

Блок 11. Проверяются соотношения между «первоначальным», «текущим» и «минимальным» временными интервалами (блоки 12, 13).

Блок 12. Если за рассматриваемый интервал времени ориентация вектора скорости ВО в «плоскости движения» меняется в пределах, не превышающих 90 , потребителю выдается информация о «параметрах движения», статистических характеристиках: ошибок их измерения и отклонений координат ВО при совершении «равновероятного маневра», сглаженное значение координат ВО.

Блок 13. При принятии решения о совершении ВО маневра с изменением ориентации вектора скорости в «плоскости движения» более чем на $90^{\circ}$ проводится уменьшение «первоначального» временного интервала завязки траектории и корректировка количества используемых отметок (оценок координат ВО) с последующим уточнением полученных оценок.

Числовые характеристики «параметров движения» и «равновероятного маневра» используются при дальнейшей фильтрации «параметров движения» и при формировании строба сопровождения ВО.

\section{Выводы}

1. В работе получен алгоритм определения параметров движения маневрирующего ВО в ЕПСК, учитывающий статистические характеристики ошибок измерения его сферических координат отдельными РЛС и переменный темп обновления информации в некогерентной системе с несинхронным обзором пространства.
2. Значения оценок параметров движения ВО (12), (14), (17)-(20), числовых характеристик ошибок их измерения (13) и отклонений координат ВО при совершении маневра в плоскости движения $(21)-(24),(27)-(28)$ в дальнейшем используются при фильтрации параметров движения ВО [5-7] в системе с несинхронным обзором пространства и переменным темпом обновления информации $\mathrm{T}_{\text {обн }}$ от нескольких неравноточных источников.

3. Полученные результаты используются в информационно-расчетных и тренажных системах на базе локальной компьютерной сети $[8,9]$, а также могут быть использованы в существующих и перспективных системах обработки РЛИ при определении параметров движения маневрирующих ВО.

\section{Список литературы}

1. Корниєнко В.В. Створення державноӥ інтегрованої системи забезпечення управління рухомими об 'єктами (зв'язок, навігація, спостереження) України - виклик XXI століття / В.В.Корниєнко, М.Ф. Бондаренко, В.Т. Гандабура, Я.С. Яиків // Наука та інновацї̈. - 2007. № 1. - Том 3. - С. 4-32.

2. Пространственно-временная обработка сигналов / И.Я. Кремер, А.И. Кремер, В.М. Петров и др. / Под ред. И.Я. Кремера. - М.: Радио и связь, 1984. - 224 с.

3. Кондратьев В.С. Многопозиционные радиотехнические системы / В.С. Кондратьев, А.Ф. Котов, Л.Н. Марков. - М.: Радио и связь, 1986. - 264 с.

4. Черняк В.С. Многопозичионная радиолокаџия / B.С. Черняк. - М.: Радио и связь, 1993. - 416 c.

5. Радиотехнические системы: Основы построения и теория. Справочник / Я.Д. Ширман, Ю.И. Лосев, Н.Н. Минервин и др. / Под ред. Я.Д. Ширмана. - М.: ЗАО «МАКВИС», 1998. -828 c.

6. Кузьмин С.3. Цифровая радиолокачия. Введение в теорию / С.3. Кузьмин. - К.: Издательство КВіЦ, 2000. $428 \mathrm{c.}$

7. Рощупкин Е.С. Оиенка прямоугольных координат цели при объединении результатов независимых первичных измерений в активной многопозиционной системе радиолокачии / Е.С. Рощупкин // Зб. наук. $п p$. Об' 'єднаного науково-дослідного інституту Збройних Сил. Х.: ОНДІ 3С, 2006. - Bun. 2(4). - C. 156-162.

8. Богдановський О.М. Очінка параметрів руху повітряних об'єктів при об'єднанні результатів незалежних первинних вимірювань в активній багатопозичійній системі радіолокачії // Системи озброєння $і$ військова техніка / О.М. Богдановський, С.В.Герасимов, Д.М. Ізосімов, C.C. Рощупкін. - 2010. - № 3 (23). - C. 110-113.

9. Рощупкин Е.C. Ошибки преобразования сферических координат радиолокачионных иелей $в$ прямоугольные / Е.С. Рощупкин // 3б. наук. пр. Об'єднаного науково-дослідного інституту Збройних Сил. - Х.: ОНДІ ЗС, 2006. - Bun. 1(3). - C. 155-161.

10. Корн Г. Справочник по математике / Г. Корн, T. Корн. - М.: Наука, 1984. -831 с.

Рецензент: М.Ю. Яковлев, д.т.н., с.н.с., Академия сухопутных войск, Львов. 
ОЦНКА ПАРАМЕТРІВ РУХУ ПОВІТРЯНИХ ОБ'ЄКТІВ, ЩО МАНЕВРУЮТЬ, В АКТИВНІЙ НЕКОГЕРЕНТНІЙ СИСТЕМІ ПРИ ОБРОБЦІ ІНФОРМАЦЇ̈ ВІД КІЛЬКОХ НЕРІВНОТОЧНИХ ДЖЕРЕЛ 3 РІЗНИМ ТЕМПОМ ОГЛЯДУ ПРОСТОРУ

С.В. Герасімов, Є.С. Рощупкін, Г.О. Федак, Я.В. Бабій

Запропоновано алгоритм визначення параметрів руху повітряного об'єкта в єдиній прямокутній системі координат при обробиі радіолокачійної інформачї, щчо отримується системою з несинхронним оглядом простору від декількох нерівноточних джерел з різним темпом оновлення інформачї.

Ключові слова: радіолокачійна інформачія, алгоритм, об'єкти, щчо маневрують.

EVALUATION OF MOTION PARAMETERS OF MANEUVERING AIR OBJECTS IN ACTIVE NON-COHERENT SYSTEM WHEN PROCESSING INFORMATION FROM SEVERAL SOURCES OF UNEQUAL ACCURACY WITH DIVERSE TEMPOES OF SPACE SURVEILLANCE

S. Herasimov, E. Roshchupkin, G. Fedak, Y. Babiy

The algorithm of determination of an air object motion operation factors in single cartesian reference system is proposed. The algorithm takes into account asynchronous air surveillance by some radars with unequal accuracy and different data rate.

Keywords: radar data, algorithm, maneuvering object.

УДК 629.1 .032

В.В. Дущенко

Національний технічний університет «Харківський політехнічний інститут», Харків

\title{
ПИТАННЯ УДОСКОНАЛЕННЯ МЕТОДОЛОГІЇ АНАЛІЗУ ТА СИНТЕЗУ СИСТЕМ ПІДРЕСОРЮВАННЯ ВІЙСЬКОВИХ ГУСЕНИЧНИХ І КОЛІСНИХ МАШИН
}

\begin{abstract}
Представлено удосконалену методологію аналізу та синтезу систем підресорювання військових гусеничних і колісних машин, щзо складається з етапів розробки вірогідної математичної моделі руху машини, функиіонально-фізичного і речовинно-польового аналізів вузлів підвіски та постановки задачі $i$ синтезу нових патентоспроможних технічних рімень і фізичних принципів дї згаданих вузлів.
\end{abstract}

Ключові слова: система підресорювання, пружні елементи, демпфіруючі пристрої, теплова напруженість, аналіз, синтез, фізичні принципи дії, технічні рішення, фізичні ефекти.

Актуальність. Система підресорювання (СП) військових гусеничних і колісних машин (ВГКМ) відіграє істотну роль у забезпеченні їх вогневої міці, захищеності та рухомості. Однак застарілі і малоефективні теоретичні підходи та методології, які на сьогодні використовуються при удосконаленні СП ВГКМ, не забезпечують можливості створення високоякісних підвісок. Внаслідок цього недосконалі СП багатьох типів машин не дозволяють повною мірою реалізовувати підвищені в процесі модернізації можливості їх удосконалених комплексів озброєння, силових установок і трансмісій. Тому актуальною науковою проблемою $\epsilon$ обгрунтування та застосування нових підходів i методологій аналізу та синтезу нових технічних рішень (ТР) і фізичних принципів дії (ФПД) вузлів СП для перспективних зразків ВГКМ.

Аналіз останніх публікацій. У роботі [1] розглянуто математичне моделювання руху військових гусеничних машин (ГМ) по нерівностях, його етапи і підходи, що використовуються, а також параметрична оптимізація торсіонної i гідропневматичної підвісок. У роботі [2] представлена найбільш повна багатофункціональна математична модель, для комплексного дослідження руху колісних машин (КМ) на різних режимах, та іï модифікація для випадку ГМ. При розробці даної моделі був використаний метод декомпозиції загальної структури на підсистеми, для кожної з яких складалася своя функціональна математична модель. Це обумовило іï складність, громіздкість і підвищення імовірності помилок. Крім того, відсутні експериментальні дані по ідентифікації зазначеної моделі та оцінці впливу тих або інших факторів, облік яких істотно ускладнює математичну модель, але не приводить до порівнянного підвищення точності розрахунків. У роботі [3] при математичному моделюванні застосовано новий 